\title{
Harmonic Passive Motion Paradigm
}

\author{
Carlo Tiseo, Sydney Rebecca Charitos, and Michael Mistry \\ ECR, Institute of Perception Action \& Behaviour, School of Informatics, University \\ of Edinburgh, Edinburgh, UK \\ E-mail: carlo.tiseo@ed.ac.uk
}

\begin{abstract}
How humans robustly interact with external dynamics is not yet fully understood. This work presents a hierarchical architecture of semi-autonomous controllers that can control the redundant kinematics of the limbs during dynamic interaction, even with delays comparable to the nervous system. The postural optimisation is performed via a non-linear mapping of the system kineto-static properties, and it allows independent control of the end-effector trajectories and the arms stiffness. The proposed architecture is tested in a physical simulator in the absence of gravity, presence of gravity, and with gravity plus a viscous force field. The data indicate that the architecture can generalise motor strategies to different environmental conditions. The experiments also verify the existence of a deterministic solution to the task-separation principle. The architecture is also compatible with Optimal Feedback Control and the Passive Motion Paradigm. The existence of a deterministic mapping implies that this task could be encoded in neural networks capable of generalisation of motion strategies to affine tasks.
\end{abstract}

\section{Introduction}

Animals can generate highly dexterous movements while dealing with kinematic redundancy, singularities, non-linear dynamics, uncertainties of interaction and signal delays. In contrast, all these scenarios are open problem in robotics, where available methods are not yet able to achieve animal-like robustness of interaction [1 15$]$. The fragility of the current model-based approaches can be related directly to the need for accurate tracking of environmental interactions to guarantee the controllers' stability [6 8], thereby hindering the development of computational models capable of explaining the nervous system capabilities.

Impedance control has been proposed as a solution to such issues by encoding compliance into our control architectures by describing the desired behaviour in terms of equivalent dynamics 1, 9 12. In general, identifying optimal movements and control is non-trivial and has resulted in the introduction of optimisation-based frameworks to identify desirable trade-offs. Thus, these methods require the exploitation of null-space projections and non-linear inverse optimisation that make them not robust to: singularities, model inaccuracy, information delay, and state discretisation 


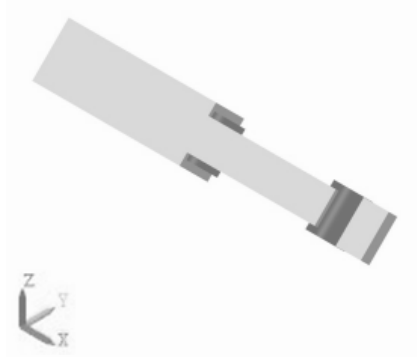

(a)

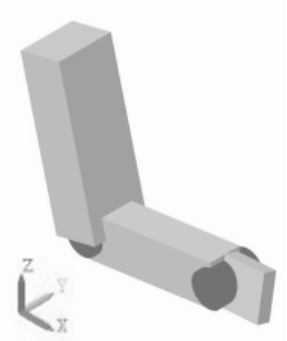

(b)

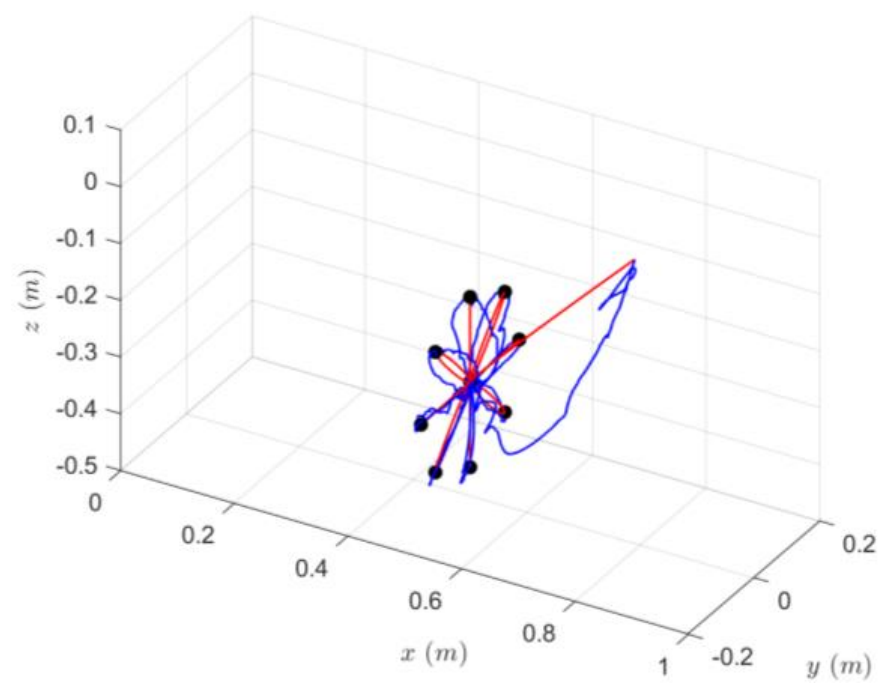

(c)

Figure 1. (a) Arm initial configuration in singularity. (b) Arm in the clock's centre. (c) In red the desired trajectory, and in blue the executed trajectory in presence of gravity and a viscous force field applied at the end-effector. The black sheres are the clock's targets.

[4, 8.13. Bioinspired learning algorithms have also been proposed to identify desirable impedance behaviours to interact with external dynamics for specific tasks [2, 3, 14,16$]$. These optimised strategies, called dynamic primitives, are defined as learned dynamic attractors required to produce the desired outcome [2, 3, 17, 18]. Recently, dynamic primitives have also been proposed as an explanation to how the nervous system deals with complex dynamic interactions [17]. Primitives are classified into three categories rhythmic, discrete, and the combined behaviours. The first category includes all the movements characterised by limit cycles; thus, encoding oscillatory motions. The discrete behaviours are typical of point attractors and describe an action such as reaching 14, 17. An example of a combined behaviour is playing drums.

Our recent work shows how adopting a hierarchical architecture of Fractal Impedance Controllers (FIC) can generate a planar human-like trajectory for upper limb reaching movements and wrist pointing trajectories. This work refines and extends that method to a 7-DoF arm (Figure 1) exploiting a recently introduced geometrical postural optimisation and introducing delays into the control loop. 


\section{Background on Motor Control}

The Central Nervous System (CNS) can accurately control the body in extremely challenging dynamics conditions. It does so without being affected by redundancy, singularities, and by the presence of noise and delays in the sensory feedback 2, 17,24 . It describes Optimal Feedback Control (OFC) 25-27] and the Passive Motion Paradigm (PMP) [19,28, 29]. The authors would like to remark that this section provides a general overview required for the contextualisation of the proposed control architecture. Thus, we refer the reader to the specialised literature for a detailed understanding of the neurophysiology of motor control, such as [21, 24, 30].

\subsection{Central Nervous System}

The CNS is organised with a hierarchical structure starting at the cortical level and reaching the Peripheral Nervous System (PNS) via the spinal cord. The hierarchical organisation is essential to deal with the delay involved in the neural transmission 2, 20, 21, 24, 30, 31]. For example, experimental results show that for balance tasks cortical potentials are delayed 200-400ms compared to en external stimulus [32], and spinal cord reflexes intervene about $100 \mathrm{~ms}$ after a perturbation occurs 33 35].

The prefrontal cortex holds the apical position in the hierarchy of the portion of the CNS that is considered involved in motor control. It is believed to attend to the control of voluntary movements and be involved in learning new skills [24]. The basal ganglia provide the reward of performing an action by assessing the associated cost of the action. The pre-motor cortex plays a key role in solving problems associated with visual feedback and the generation of a task-space movement strategy 24, 30, 31]. The motor cortex is involved in controlling the movement dynamics, based on the proprioceptive feedback $2,24,31]$. The parietal cortex processes the state estimation for both the proprioceptive and the visual feedback, and it is strictly interconnected with the internal models allocated in the cerebellum $2,24,31$.

The commands issues from the motor cortex reach the Central Pattern Generators in the spinal cord, which is in charge of synchronised oscillatory movements, and they receive inputs from the motor cortex, cerebellum, and the afferent sensory information arriving from the periphery $2,24,36,37]$. The spinal cord is also the loci of the spinal cord reflexes that are the first intervention strategy in the spinal cord. These reflexes provide the first centralised response to proprioceptive feedback $2,20,35,36]$. It is worth noting that considering that the delays of these reflexes are in the order of $100 \mathrm{~ms}$, the modulation of the muscle-skeletal system dynamics is essential to movement stability by filtering high-frequency perturbations.

\subsection{Optimal Feedback Control 6 Separation Principle}

The OFC exploits optimal control theory to explain how the nervous systems optimise human movements, stabilise the task, and control the redundant degrees of freedoms 
[25, 26, 30]. The OFC describes the nervous systems as a model-based continuous feedback controller, which exploits multiple cost functions for different tasks [27, 38]. The framework was successfully used to model the separation principle by classifying these controllers for dynamic and static tasks [19,27, 29, 38]. Dynamic controllers deal with the interaction with the dynamics forces (inertial and velocity-dependent forces), and the static controllers compensate for the static forces (elastic fields and gravity) 27]. The model has been proven capable of mimicking human behaviour, capturing how motor control adjusts to sensory feedback during task execution [27, 38]. However, the nervous system deals with the non-holonomic manifolds generated from their joint-space formulation, which is not compatible with the holonomic characteristics experimentally observed in humans and described by Donders'Law [19,29,30].

\subsection{Equilibrium Point Hypothesis \& Passive Motion Paradigm}

A well-known theory for movement generation is the Equilibrium Point Hypothesis (EPH) which describes the generation of reaching movements as a gradual shift of the equilibrium point $[2,19,28,29,39]$. Further evidence also shows that the brain process separately deals with postural control (i.e., static forces) and motion dynamics, known as the separation principle. The PMP is a control framework that utilises this principle $[2,4,5,19,29]$. The $\lambda_{0}$-PMP is a recently proposed extension of this framework. It relies on a viscous force field to produce a constraint that limits the projections of some of the joint torques in the task space while optimising posture in the manipulator null-space [19,29]. Other studies show that a similar result can be obtained by superimposing multiple task-space conservative non-linear impedance controllers to generate virtual mechanical constraints along the kinematic chain. This approach relies on a Fractal Impedance Controller (FIC) to define a non-linear impedance controller around the desired state [4, 5, 40]. Differently from the $\lambda_{0}$-PMP this latest approach has the advantage that it does not rely on identifying a damping component and matrix inversions, which is taken over by the introduction of a non-linear spring [5]. Therefore, the controller energy is a path independent potential field (i.e., holonomic); thus, respecting Donders's Law [5, 19,29].

\section{Harmonic Passive Motion Paradigm}

The Harmonic Passive Motion Paradigm (H-PMP) is a controller architecture describing communication from the motor cortex to its related muscle groups. The H-PMP can integrate haptic control, but this functionality is omitted for simplicity in this work. The H-PMP architecture is schematised in Figure 2.

\subsection{Cartesian-Space Harmonic Trajectory Planning}

Cartesian trajectory planning is traditionally associated to a Cost-To-Go of the basal ganglia, usually implemented via optimisation to generate minimum jerk trajectories. 


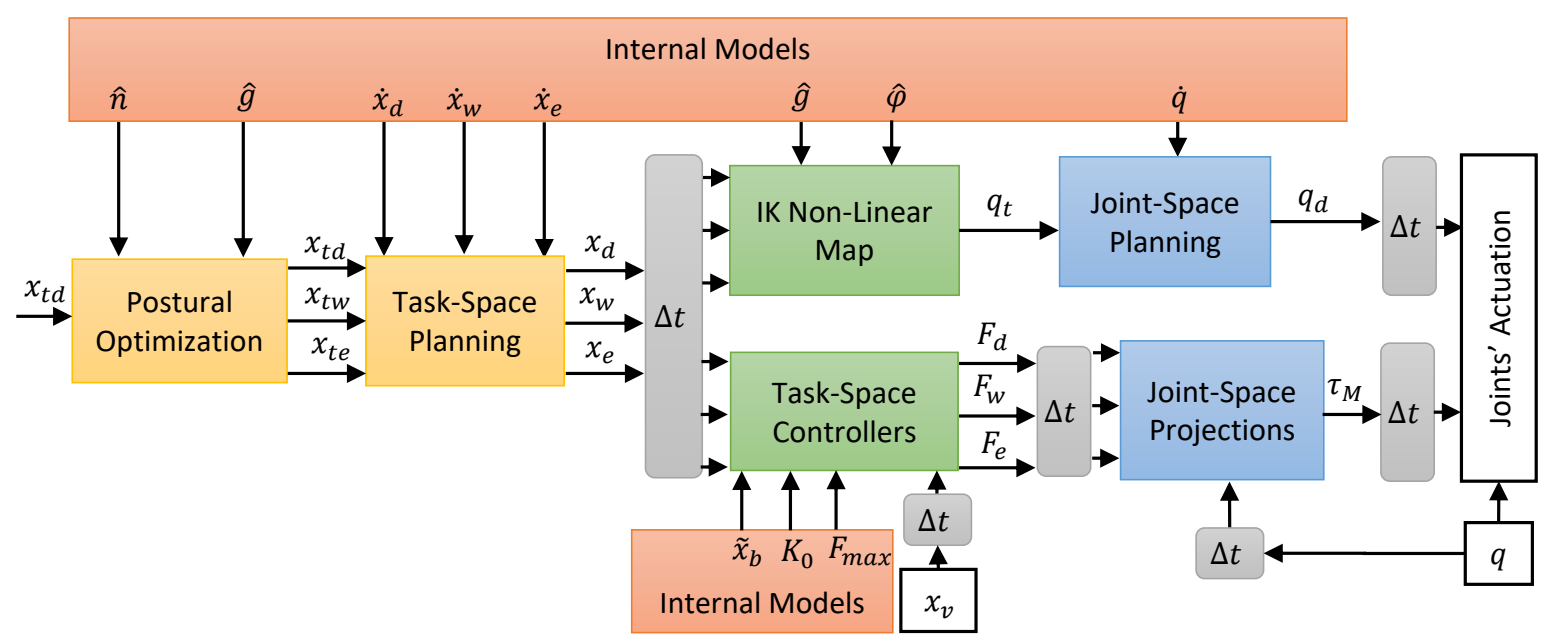

Figure 2. The H-PMP architecture starts from the postural optimisation of the limb. It takes into input the end-effector target for the hand $x_{\mathrm{td}}$, and an internal model of the task in terms of motion plane $\hat{n}$ and grasp direction $\hat{g}$. The postural optimisation generates targets for the wrist $\left(x_{\mathrm{w}}\right)$ and the elbow $\left(x_{\mathrm{e}}\right)$. These three targets are the input of the task-space planners, which based on the desired velocities associated with the task's internal model $\left(\dot{x}_{\mathrm{d}}, \dot{x}_{\mathrm{w}}\right.$ and $\left.\dot{x}_{\mathrm{e}}\right)$ generate the limbs' trajectories. The limbs' trajectories are delayed on an interval $\Delta t$ and, subsequently, passed as input to both the IK and the task-space controllers. The IK is solved using a non-linear map which depends from $\hat{g}, \dot{x}_{\mathrm{d}}, \dot{x}_{\mathrm{w}}, \dot{x}_{\mathrm{e}}$, and the unit vector for wrist pronosupination $\hat{\phi}$, and its output is passed to the joints' trajectory planners to derive the desired joints' trajectories $\left(q_{\mathrm{d}}\right)$. The three task space controllers input are the desired end-effector trajectories, their delayed visual feedback $\left(x_{\mathrm{v}}\right)$, and the models' parameters of the non-linear impedance $\left(\tilde{x}_{\mathrm{b}}, K_{0}\right.$ and $\left.F_{\max }\right)$. The output forces are passed to derive the desired joints' torques $\left(\tau_{\mathrm{M}}\right)$, which requires the knowledge of the joints' configuration $q$. Lastly, the $q_{\mathrm{d}}$ and $\tau_{\mathrm{M}}$ are the input of the joints' actuation modelling the coordinated actions of the muscle groups.

We have recently proposed a planner which employs the FIC to generate harmonic trajectories that have been proven to capable of generating human-like kinematics on planar arm kinematics in [4,5, 13, 41]. Harmonic trajectories are preferable because they require a lower peak power compared to minimum jerk trajectories for generating the same average velocity [4,5]. An independent planner for each of the task-space DoF is implemented as an Model Predictive Controller (MPC) per unit of mass. The desired trajectory is computed via the integration of the desired acceleration $\left(\ddot{x}_{\mathrm{d}}\right)$ output from the controller.

$$
x_{\mathrm{d}}=\iint_{t_{0}}^{t} \ddot{x}_{\mathrm{d}}(t) d t^{2}
$$


where $t_{0}$ is the initial time, and $t$ is the current time. The acceleration is derived from the following equation:

$$
\ddot{x}_{\mathrm{d}}(t)= \begin{cases}\operatorname{sign}\left(\tilde{x}_{\mathrm{t}}\right) \min \left(\frac{K}{M_{\mathrm{d}}}\left|\tilde{x}_{\mathrm{t}}\right|, a_{\mathrm{max}}\right), & \operatorname{Div} \\ \frac{2 A_{\max }}{\tilde{x}_{\mathrm{T} 0}}\left(x_{\mathrm{d}}(t-1)-\frac{\tilde{x}_{\mathrm{T} 0}}{2}\right), & \text { Conv }\end{cases}
$$

where $\tilde{x}_{\mathrm{t}}=x_{\mathrm{t}}-x_{\mathrm{d}}(t-1), A_{\max }=2 v_{\max }^{2} / \tilde{x}_{\mathrm{T} 0}$ is the acceleration computed at the maximum displacement $\left(\tilde{x}_{\mathrm{T} 0}\right)$ reached in the previous divergence phase, and $v_{\max }=$ $1.596 v_{\mathrm{d}}$ is the maximum velocity of the harmonic trajectory generating the desired velocity $v_{\mathrm{d}}$.

\subsection{Arm Postural Optimisation}

The arm postural optimisation and IK exploit a recently introduced geometrical solution, described by one of the functions of the motor cortex. We have chosen this approach because it generates a deterministic non-linear mapping robust to singularities between the task-space and the joint-space exploiting the kineto-static duality [42], and could be performed by the nervous system relying on an internal model present in the cerebellum. The definition of the optimal posture can be identified by aligning the arm plane of motion with the task dynamics, leading to manipulability properties equivalent to a planar 3-link arm 42].

Let us define the following unit vectors requires by the formulations of the postural optimisation and inverse kinematics:

- $\hat{x}_{\mathrm{e}}$ is the direction of the arm link

- $\hat{x}_{\mathrm{w}}$ is the direction of the wrist vector

- $\hat{x}_{\mathrm{d}}$ is the direction of end-effector vector

- $\hat{g}$ is the grasp direction

- $\hat{\phi}$ is the direction controlling the hand-pronosupination

- $l_{\mathrm{a}}$ is the arm length

- $l_{\mathrm{fa}}$ is the forearm length

- $l_{\mathrm{h}}$ is the hand length

- $\hat{n}$ as the unit vector orthogonal to the task's plane 
The postural optimisation equations for a left-arm can now be written as follows:

$$
\begin{aligned}
& x_{\mathrm{w}}=x_{\mathrm{d}}-l_{h} \hat{g} \\
& \vec{h}=\hat{x}_{\mathrm{w}} \times \hat{n} \\
& \hat{h}=\frac{\vec{h}}{\|\vec{h}\|} \\
& k=\frac{l_{\mathrm{fa}}}{l_{\mathrm{a}}} \\
& l_{\mathrm{a}}^{\prime}=\frac{\left\|x_{\mathrm{w}}\right\|}{2}+\frac{l_{\mathrm{a}}^{2}\left(1-k^{2}\right)}{2\left\|x_{\mathrm{w}}\right\|} \\
& \mu=\sqrt{l_{\mathrm{a}}^{\prime}-l^{2}} \\
& x_{\mathrm{e}}=\left\{\begin{array}{l}
-\mu \hat{h}+l_{\mathrm{a}}^{\prime} \hat{x}_{\mathrm{w}}, \quad\left(\hat{h}_{2}<0 \& x_{\mathrm{d}} \geq 0\right) \wedge\left(\hat{h}_{2} \leq 0 \& x_{\mathrm{d}} \leq 0\right) \\
+\mu \hat{h}+l_{\mathrm{a}}^{\prime} \hat{x}_{\mathrm{w}}, \text { Otherwise }
\end{array}\right.
\end{aligned}
$$

The equation for the right arm can be easily obtained by inverting the signs of $\mu \hat{h}$. The joint angle can be derived from the following equations:

$$
\begin{aligned}
& q_{\mathrm{t} 1}=\arctan \left(\hat{x}_{\mathrm{e}} \cdot R_{1 y}^{0}, \hat{x}_{\mathrm{e}} \cdot R_{1 x}^{0}\right) \\
& q_{\mathrm{t} 2}=\arctan \left(\hat{x}_{\mathrm{e}} \cdot R_{2 y}^{1}, \hat{x}_{\mathrm{e}} \cdot R_{2 x}^{1}\right) \\
& q_{\mathrm{t} 3}=\arctan \left(\hat{x}_{\mathrm{e}} \cdot R_{3 y}^{2}, \hat{x}_{\mathrm{e}} \cdot R_{3 x}^{2}\right) \\
& q_{\mathrm{t} 4}=\arctan \left(\left(\hat{x}_{\mathrm{w}}-\hat{x}_{\mathrm{e}}\right) \cdot R_{4 y}^{3},\left(\hat{x}_{\mathrm{w}}-\hat{x}_{\mathrm{e}}\right) \cdot R_{4 x}^{3}\right) \\
& \alpha=\hat{g} \times(\hat{\phi} \times \hat{g}) \\
& q_{\mathrm{t} 5}=\arctan \left(\alpha \cdot R_{5 y}^{4}, \alpha \cdot R_{5 x}^{4}\right) \\
& q_{\mathrm{t} 6}=\arctan \left(\hat{g} \cdot R_{6 y}^{5}, \hat{g} \cdot R_{6 x}^{5}\right) \\
& q_{\mathrm{t} 7}=\arctan \left(\hat{g} \cdot R_{7 y}^{6}, \hat{g} \cdot R_{7 x}^{6}\right)
\end{aligned}
$$

where $q_{\mathrm{t} i}$ is the $i^{\text {th }}$ joint angle of the target, $R_{i}$ is the rotation matrix of the base from of the $i^{\text {th }}$ link, and $R_{i}^{i-1}$ is the base frame of the $i^{\text {th }}$ joint.

\subsection{Joint-Space Harmonic Trajectory Planning}

Joint space planners are based on a variation of the model used for the Task-Space planning in subsection 3.1. The maximum angular acceleration and velocity are descriptive of the maximum mechanical characteristics of each joint.

$$
q_{\mathrm{d} i}=\iint_{t_{0}}^{t} \ddot{q}_{\mathrm{d} i}(t) d t^{2}
$$

The acceleration is derived from the following equation:

$$
\ddot{q}_{\mathrm{d}}(t)= \begin{cases}\operatorname{sign}\left(\tilde{q}_{\mathrm{t} i}\right) \min \left(\frac{K}{M_{\mathrm{d}}}\left|\tilde{q}_{\mathrm{t} i}\right|, \dot{\omega}_{\mathrm{max}-i}\right), & \text { Div } \\ \frac{2 \dot{\omega}_{\mathrm{max}-i}}{\tilde{q}_{\mathrm{T} 0 i}}\left(q_{\mathrm{d} i}(t-1)-\frac{\tilde{q}_{\mathrm{t} i}}{2}\right), & \text { Conv }\end{cases}
$$


where $\tilde{q}_{\mathrm{ti}}=q_{\mathrm{ti}}-q_{\mathrm{di}}(t-1), \dot{\omega}_{\max -i}=2 \omega_{\max -i}^{2} / \tilde{q}_{\mathrm{T} 0 i}$ is the maximum acceleration that can be computed known the maximum angular velocity $\left(\omega_{\max -i}\right)$ and the maximum displacement reached in the previous divergence phase $\left(\tilde{q}_{\mathrm{T} 0 i}\right)$.

\subsection{Task-Space Interaction Control}

The Task-Space interaction is a variation superimposition of multiple Fractal Impedance controllers based on the methods tested in [4, 40]. Task-space controllers are assigned to control the pronosupination and each of the 3-links composing the arm. They will prescribe the effort along the kinematic chain determining the response to external dynamics and mechanical losses. The projection of such information in joint space will be explained in the next section.

The FIC effort (i.e, force or torque) is computed according to the following equation:

$$
F_{\xi}(\tilde{x})=\left\{\begin{array}{cc}
F_{\mathrm{c}}(\tilde{x}), & \text { Divergence } \\
\frac{2 F_{\mathrm{c}}\left(\tilde{x}_{\max }\right)}{\tilde{x}_{\max }}\left(\tilde{x}-\frac{\tilde{x}_{\max }}{2}\right) & \text { Convergence }
\end{array}\right.
$$

where $F_{\mathrm{c}}$ is a generic continuous and upper-bounded force profile, $\tilde{x}$ is the pose error, $\tilde{x}_{\max }$ is maximum pose error reached during the previous divergence phase [4, 11, 41]. The chosen force profile is single sigmoidal force saturation enclosing a region of linear impedance around the desired pose, based on the formulation proposed in [4, 41].

$$
F_{\mathrm{c}}= \begin{cases}K_{0} \tilde{x}=K_{0}\left(x_{\mathrm{d}}(t)-x\right), & \tilde{x} \leq 0.95 \tilde{x}_{\mathrm{b}} \\ \frac{\Delta F}{2}\left(\tanh \left(\frac{\tilde{x}-\tilde{x}_{\mathrm{b}}}{S \tilde{x}_{b}}+\pi\right)+1\right)+F_{0}, & \mathrm{o} / \mathrm{w}\end{cases}
$$

where $K_{0}$ is the constant stiffness, $\tilde{x}$ is the end-effector pose error, $\Delta F=F_{\max }-F_{0}$, $F_{0}=0.95 K_{0} \tilde{x}_{\mathrm{b}}$, and $S=0.008$ controls the saturation speed to be completed between the last $5 \%$ of $\tilde{x}_{\mathrm{b}}$.

\subsection{Joint-Space Projections}

Joint coordination is believed to be addressed via the central pattern generators of the spinal cord; which is also responsible for the control of the reflexes due to its proximity to the periphery. Similarly, we have allocated the logic for the torque command generation at a low level, to reduce the delay in the transmission of postural information to be utilised to compute the Jacobian transpose. It is worth noting that although we have omitted the reflex in this work for simplicity, they could be implemented using the haptic algorithm presented in [41]. The desired forces $F_{\xi}$ from Equation 7 are computed for the elbow $F_{\mathrm{e}}$, the wrist $F_{\mathrm{w}}$, and the end-effector $F_{\mathrm{d}}$ before projecting them in the joint space using the respective geometric Jacobians $\left(J_{\mathrm{e}}, J_{\mathrm{w}}\right.$ and $\left.J_{\mathrm{d}}\right)$.

$$
\begin{aligned}
& \tau_{e}=\left|J_{\mathrm{e}}(q)^{\mathrm{T}}\left(F_{\mathrm{e}}^{\mathrm{T}} 0_{1 \times 3}\right)^{\mathrm{T}}\right| \\
& \tau_{w}=\left|J_{\mathrm{w}}(q)^{\mathrm{T}}\left(F_{\mathrm{w}}^{\mathrm{T}} 0_{1 \times 3}\right)^{\mathrm{T}}\right| \\
& \tau_{h}=\left|J_{\mathrm{d}}(q)^{\mathrm{T}}\left(\begin{array}{ll}
F_{\mathrm{d}}^{\mathrm{T}} & 0_{1 \times 3}
\end{array}\right)^{\mathrm{T}}\right|
\end{aligned}
$$


Meanwhile the pronosupination control directly generates a torque $\tau_{\text {PS }}$ acting only on the fifth joint controlling the pronosupination. The four torques vectors are then combined to control the maximum torque of the joints' actuation presented in the following section.

$$
\tau_{\mathrm{M}}=\left(\begin{array}{l}
\max \left(\tau_{\mathrm{e} 1}, \tau_{\mathrm{w} 1}, \tau_{\mathrm{d} 1}\right) \\
\max \left(\tau_{\mathrm{e} 2}, \tau_{\mathrm{w} 2}, \tau_{\mathrm{d} 2}\right) \\
\max \left(\tau_{\mathrm{w} 3}, \tau_{\mathrm{d} 3}\right) \\
\max \left(\tau_{\mathrm{w} 4}, \tau_{\mathrm{d} 4}\right) \\
\max \left(\tau_{\mathrm{d} 5},\left|\tau_{\mathrm{PS}}\right|\right) \\
\tau_{\mathrm{d} 6} \\
\tau_{\mathrm{d} 7}
\end{array}\right)
$$

where $\tau_{\text {ei }}, \tau_{\text {wi }}, \tau_{\text {di }}$ are the $i^{\text {th }}$ components of the respective vectors. This strategy guarantees the maximum rigidity of the arm based on the output of the 4 independent task-space controllers (3 links plus wrist pronosupination).

\subsection{Joints' Controller}

The proposed control architecture describes the overall joint actuation resulting from the muscle activity in each joint. Therefore, each of the joints are controlled using a fractal impedance controller generating the following joint command:

$$
\tau_{i}=\left\{\begin{array}{cc}
\tau_{\mathrm{c}-i}\left(\tilde{q}_{i}\right), & \text { Divergence } \\
\frac{2 \tau_{\mathrm{c}-i}\left(\tilde{q}_{i}\right)}{\tilde{q}_{\text {max } \mathrm{i}}}\left(\tilde{q}_{i}-\frac{\tilde{q}_{\text {max }-\mathrm{i}}}{2}\right) & \text { Convergence }
\end{array}\right.
$$

where $\tau_{\mathrm{c}-i}$ is a continuous upper-bounded torque profile, $\tilde{q}_{i}=\tilde{q}_{\mathrm{d}-i}-q_{i}$ is the error between the current and the desired angle, $\tilde{q}_{\text {max }-i}$ is the maximum angular error reached during the previous divergence phase. The torque profile $\left(\tau_{\mathrm{c}-i}\right)$ used in this paper is:

$$
\tau_{\mathrm{c}-i}\left(\tilde{q}_{i}\right)=\left\{\begin{array}{cl}
\frac{\tau_{\mathrm{M}-i}}{\delta q_{i}} \tilde{q}_{i}, & \left|\tilde{q}_{i}\right| \leq \delta q_{i} \\
\tau_{\mathrm{M}-i}, & \delta q_{i}<\left|\tilde{q}_{i}\right| \leq k \delta q_{i} \\
\tau_{\max -i}, & \left|\tilde{q}_{i}\right| \geq k \delta q_{i}
\end{array}\right.
$$

where $\tau_{\max -i}$ is the maximum actuation torque, $\delta q_{i}$ is angular accuracy, and $k$ a scaling factor. The chosen formulation allows to model the muscular activities as a change of the equilibrium position of a potential field generated by the agonist and antagonist muscles. This choice is based on theoretical and experimental data showing that the antagonist muscles group are responsible for the modulation of the joints' impedance [4, 43].

\section{Validation Method}

The H-PMP has been validated on two arm simulators in Simulink (Mathworks, USA) developed using the Simscape library for physics simulations, using the ode-45 solver 
with a maximum time step of $1 \mathrm{~s}$. The first simulator evaluates the proposed method without accounting for the information delay. The second simulator evaluates the HPMP performance in the presence of delays comparable with the nervous system without using a state estimator.

\subsection{Arm Model}

The arm is modelled as a 7-dof manipulator with a spherical-revolute-spherical joint configuration. The shoulder joint has a ZYX configuration; meanwhile, the wrist joint has a XYZ configuration. The lengths of the arm, forearm and hand are $l_{\mathrm{a}}=0.37 \mathrm{~m}$, $l_{\mathrm{a}}=0.32 \mathrm{~m}$ and $l_{\mathrm{h}}=0.1 \mathrm{~m}$, respectively . Their masses are $M_{\mathrm{a}}=4.1 \mathrm{~kg}, M_{\mathrm{fa}}=2.4 \mathrm{~kg}$, and $M_{\mathrm{h}}=1 \mathrm{~kg}$. Each joint has a friction coefficient of $K_{\mu}=0.5 \mathrm{Nms} / \mathrm{deg}$.

4.1.1. Planner \&6 Controller Parameters The task-space planners have been set with a reference velocity $v_{\mathrm{d}}=0.5 \mathrm{~m} \mathrm{~s}^{-1}$ with a minimum $A_{\max }=0.1 \mathrm{~m} / \mathrm{s}^{2}$, and $K / M_{\mathrm{d}}=4000 \mathrm{~s}^{-2}$ The joint-space planners have been set with a reference velocity $\omega_{\mathrm{d}}=0.1 \pi \mathrm{rad} \mathrm{s}^{-1}$, and $K / M_{\mathrm{d}}=4000 \mathrm{~s}^{-2}$. The pronosupination unit vector $\hat{\phi}$ is fixed at $\left(\begin{array}{lll}0 & 0 & 1\end{array}\right)^{T}$ for all experiments. Similarly the grasp unit vector $\hat{g}$ is also been kept constant at $\left(\begin{array}{lll}1 & 0 & 0\end{array}\right)^{T}$ for all the experiment.

The parameters of the hand's task-space controller are $K_{0}=10000 \mathrm{~N} \mathrm{~m}^{-1}, \tilde{x}_{\mathrm{b}}=$ $5 \mathrm{~mm}$, and $F_{\max }=200 \mathrm{~N}$. The parameters of the wrist's task-space controller are $K_{0}=6000 \mathrm{~N} \mathrm{~m}^{-1}, \tilde{x}_{\mathrm{b}}=5 \mathrm{~mm}$, and $F_{\max }=200 \mathrm{~N}$. The parameters of the elbow's taskspace controller are $K_{0}=1000 \mathrm{~N} \mathrm{~m}^{-1}, \tilde{x}_{\mathrm{b}}=10 \mathrm{~mm}$, and $F \max =100 \mathrm{~N}$. The torsional reference pronosupination torque $\tau_{P S}$ has been kept constant at $15 \mathrm{Nm}$, being phi constant. The parameters of the joints' controllers are $k=5, \delta q_{i}=0.0175 \operatorname{rad} \forall i \in[1,7]$, and $\tau_{\text {max }}=\left(\begin{array}{llll}300300150150505050\end{array}\right)^{T} \mathrm{~N} \mathrm{~m}$.

4.1.2. Information Delays The $\Delta t$ used between the task-space planning and the IK against the task-space controller in Figure 2 is $0.1 \mathrm{~ms}$. The delay introduced between the the joint-space planning and the joints' actuation is also $0.1 \mathrm{~ms}$. The delay between the task-space controllers and the joint-space projections is $0.04 \mathrm{~ms}$. Consequently, the delay between the latter component and the joints' actuation is $0.06 \mathrm{~ms}$. The delay of the visual feedback $\left(x_{\mathrm{v}}\right.$ in Figure 2 ) has been set to $0.2 \mathrm{~ms}$, and the delay for the proprioceptive feedback to the joint-space projections is $0.04 \mathrm{~ms}$.

\subsection{Clock Experiment}

The clock experiment experiment is a common tool used for studying human movements and the motor control framework [19, 27, 29, 44]. The centre of the clock is in $(0.500 \quad 0-0.300) \mathrm{m}$ and has a radius of $15 \mathrm{~cm}$. Thus, the coordinates of the 8 targets are: $(0.5000 .106-0.406) \mathrm{m},(0.5000-0.450) \mathrm{m},(0.500-0.106-0.406) \mathrm{m}$, $(0.500-0.150-0.300) \mathrm{m},(0.500-0.106-0.194) \mathrm{m},(0.500 \quad 0 \quad-0.150) \mathrm{m}$, $(0.5000 .106-0.194) \mathrm{m}$, and $(0.5000 .150-0.300) \mathrm{m}$. 
The experiment has been performed three times for each simulator to evaluate the effect on external force fields on the arm stability, and the tracking performances. All the simulations started from a fully extended posture aligned with the x-axis. The three conditions are: without gravity, with gravity, and with gravity plus viscous force field applied to the hand's end-effect.

The formulation of the viscous force field is:

$$
F_{\mathrm{v}}=\left(\begin{array}{ccc}
0 & 100 & -100 \\
-100 & 0 & 100 \\
100 & -100 & 0
\end{array}\right) \dot{x}_{e}
$$

\subsection{Data Analysis}

The Mean Absolute Error (MAE) and the Root Mean Square Error (RMSE) are computed for the trajectories of the task-space and joint-space controllers. The MAE is used to evaluate the average tracking accuracy. Meanwhile, the RMSE quantifies the impact of sporadic large errors (i.e., outliers) on the tracking performance.

The mean and the standard deviation of the ratio between the average and the peak velocity $(C=\max (\dot{x}) / \operatorname{mean}(\dot{x}))$ of the task-space trajectories have been computed and compared with the human range of $C_{\mathrm{H}}=1.805 \pm 0.153,4,45$ to validate the similarity with human movements.

\section{Results}

The planned and the executed trajectories in Figure 3 show that the arm always reaches, but there is a reduction of the tracking accuracy with the perturbation increase. The MAE and the RMSE are reported in Table 1. The RMSE is higher, suggesting the sporadic occurrence of large errors during the trajectory tracking. The two tracking errors increase when introducing the delays, which is expected due to the increased time-shift between the issuing of the command and its execution. The data also show that the accuracy at the elbow end-effector is constrained to $\tilde{x}_{\mathrm{b}}$, but it degenerates beyond $\tilde{x}_{\mathrm{b}}$ for both the wrist and the hand controllers. Thus, suggesting that it might be related to the accumulation of the joints' position errors and can be compensated by introducing a state estimator. The MSA and the RMSA in the joint-space (Table 2) show that the errors of the first five joints are always constrained within the accuracy set in the controllers. Meanwhile, the sixth and the seventh joints are consistently beyond suggesting a need for an increase in the system stiffness to achieve the desired accuracy. The joints' errors also confirm that the task-space accuracy can be improved by introducing a state estimator to compensate for the systematic error.

The distribution of the $C$ values for the planned trajectory (Figure 4 ) are consistent with the value of the harmonic trajectories $\left(C_{\mathrm{HT}}=1.596\right)$, and the distribution have little variance which does not overlap with the interval measured for human movements $C_{\mathrm{H}}=1.805 \pm 0.153[39$. Figure 5 presents the data for the executed trajectories 

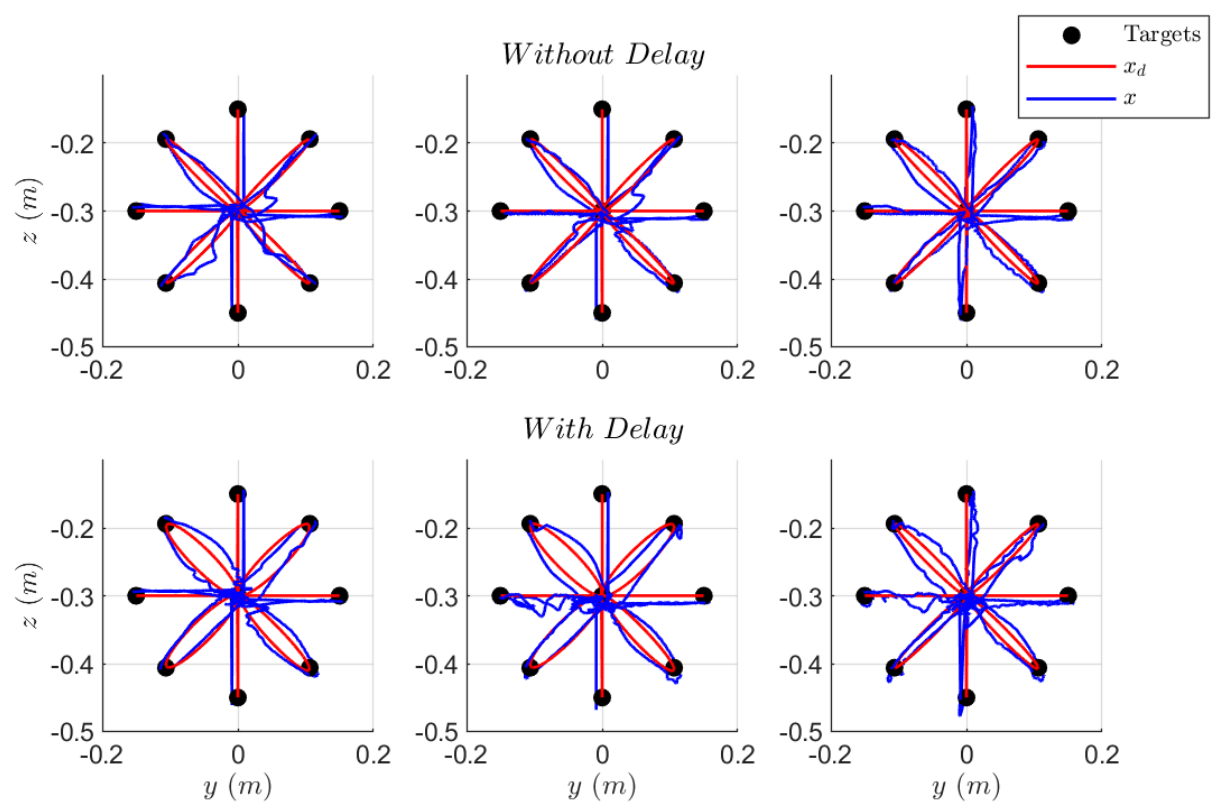

Figure 3. Reaching trajectories in the three considered conditions for the H-PMP with and without delay. The trajectories in the absence of gravity are on the left. The data recorded for the gravity scenario are in the centre. The trajectories for the scenario of gravity plus viscous force field are on the right. We observe a general reduction of accuracy moving from right to left.

showing a substantial overlap with $C_{\mathrm{H}}$. The One-Way ANOVA has shown no significant difference in the $\mathrm{C}$ values recorded in the six experimental conditions. The test $p$-value is 0.9442. The data considered as belonging to a single population are compared with the human data in Figure 6, which confirms the overlap. The data in Figure 5 and Figure 6 show that the data from our simulation show a higher variability compared to both the human data and our preliminary results obtained for a 3-link planar arm [4]. It indicates that this variability could be related to sudden velocity peaks due to perturbation or not optimal coordination between the different architectural components. This analysis is confirmed by the data on the end-effector tangential velocity in Figure 7, showing that the executed trajectories have an expected trend of a 10 to $15 \%$ increase in peak velocity compared to the planned trajectories. However, the data also show that there are episodically peaks that are substantially higher, which supports the hypothesis mentioned above that they are related to local conditions due to perturbations and lack of coordination.

\section{Discussion}

The results show that the H-PMP can generate human-like movements without the need for numerical optimisation and regression of the controller parameters from the human data. The architecture is also robust to the communication delay intrinsic to neuronal 


\begin{tabular}{|c|c|c|c|}
\hline & $\operatorname{MAE}\left(\tilde{x}_{\mathrm{e}}\right)[\mathrm{mm}]$ & $\operatorname{MAE}\left(\tilde{x}_{\mathrm{w}}\right)[\mathrm{mm}]$ & $\operatorname{MAE}\left(\tilde{x}_{\mathrm{d}}\right)[\mathrm{mm}]$ \\
\hline No Gravity & $\left(\begin{array}{lll}0.3 & 0.2 & 1.1\end{array}\right)^{T}$ & $(6.82 .33 .4)^{T}$ & $(6.82 .33 .4)^{T}$ \\
\hline Gravity & $\left(\begin{array}{lll}1.6 & 0.4 & 1.5\end{array}\right)^{T}$ & $\left(\begin{array}{lll}6.5 & 1.3 & 4.5\end{array}\right)^{T}$ & $\left(\begin{array}{lll}6.5 & 1.6 & 4.5\end{array}\right)^{T}$ \\
\hline Viscous Field & $\left(\begin{array}{lll}1.7 & 0.6 & 1.5\end{array}\right)^{T}$ & $\left(\begin{array}{lll}6.5 & 1.6 & 4.7\end{array}\right)^{T}$ & $\left(\begin{array}{lll}6.5 & 1.6 & 4.7\end{array}\right)^{T}$ \\
\hline No Gravity \& Delay & $(1.53 .61 .8)^{T}$ & $\left(\begin{array}{llll}7.5 & 6.0 & 6.0\end{array}\right)^{T}$ & $\left(\begin{array}{llll}7.5 & 6.0 & 6.0\end{array}\right)^{T}$ \\
\hline Gravity \& Delay & $(2.53 .72 .1)^{T}$ & $(7.56 .17 .5)^{T}$ & $(7.56 .17 .5)^{T}$ \\
\hline \multirow[t]{2}{*}{ Viscous Field \& Delay } & $\left(\begin{array}{lll}2.7 & 3.8 & 2.1\end{array}\right)^{T}$ & $(6.86 .48 .0)^{T}$ & $\left(\begin{array}{llll}6.8 & 6.4 & 8.0\end{array}\right)^{T}$ \\
\hline & $\operatorname{RMSE}\left(\tilde{x}_{\mathrm{e}}\right)[\mathrm{mm}]$ & $\operatorname{RMSE}\left(\tilde{x}_{\mathrm{w}}\right)[\mathrm{mm}]$ & $\operatorname{RMSE}\left(\tilde{x}_{\mathrm{d}}\right)[\mathrm{mm}]$ \\
\hline No Gravity & $\left(\begin{array}{llll}0.8 & 0.6 & 3.0\end{array}\right)^{T}$ & $(10.65 .65 .3)^{T}$ & $(10.58 .97 .8)^{T}$ \\
\hline Gravity & $\left(\begin{array}{lll}2.1 & 0.7 & 3.1\end{array}\right)^{T}$ & $(10.52 .86 .0)^{T}$ & $(10.58 .09 .6)^{T}$ \\
\hline Viscous Field & $(2.21 .33 .2)^{T}$ & $(10.42 .46 .3)^{T}$ & $(10.47 .29 .4)^{T}$ \\
\hline No Gravity \& Delay & $\left(\begin{array}{llll}3.6 & 6.6 & 3.6\end{array}\right)^{T}$ & $(11.58 .68 .3)^{T}$ & $\left(\begin{array}{lll}11.5 & 11.9 & 8.4\end{array}\right)^{T}$ \\
\hline Gravity \& Delay & $(4.16 .63 .8)^{T}$ & $(11.48 .89 .5)^{T}$ & $\left(\begin{array}{lll}11.4 & 12.0 & 10.9\end{array}\right)^{T}$ \\
\hline Viscous Field \& Delay & $(4.56 .63 .8)^{T}$ & $\left(\begin{array}{lll}10.4 & 8.9 & 10.2\end{array}\right)^{T}$ & $\left(\begin{array}{lll}10.4 & 12.3 & 11.4\end{array}\right)^{T}$ \\
\hline
\end{tabular}

Table 1. The MEA and RMSE for the task-space controllers.

\begin{tabular}{|c|c|}
\hline & $\operatorname{MAE}(\tilde{q})[\mathrm{mrad}]$ \\
\hline No Gravity & $\left(\begin{array}{lllllll}2.2 & 0.0 & 8.3 & 6.3 & 2.1 & 69.4 & 76.4\end{array}\right)^{T}$ \\
\hline Gravity & 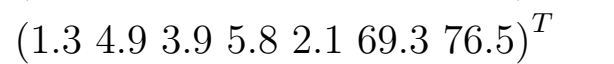 \\
\hline Viscous Field & 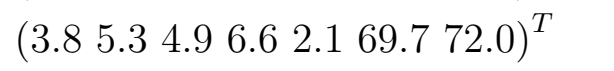 \\
\hline No Gravity \& Delay & 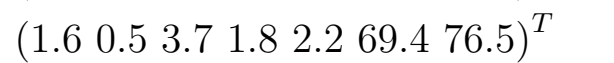 \\
\hline Gravity \& Delay & $(1.44 .73 .74 .92 .270 .576 .6)^{T}$ \\
\hline \multirow[t]{2}{*}{ Viscous Field \& Delay } & 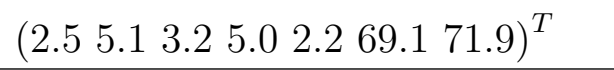 \\
\hline & $\operatorname{RMSE}(\tilde{q})[\mathrm{mrad}]$ \\
\hline No Gravity & $\left(\begin{array}{llllllll}7.0 & 0.7 & 20.7 & 11.8 & 3.4 & 72.7 & 79.6\end{array}\right)^{T}$ \\
\hline Gravity & $(2.96 .19 .99 .73 .474 .779 .6)^{T}$ \\
\hline Viscous Field & $(10.46 .712 .310 .63 .473 .976 .2)^{T}$ \\
\hline No Gravity \& Delay & $\left(\begin{array}{l}4.41 .09 .33 .63 .572 .7 \quad 79.7\end{array}\right)^{T}$ \\
\hline Gravity \& Delay & $(3.67 .111 .29 .03 .575 .179 .7)^{T}$ \\
\hline Viscous Field \& Delay & $(5.58 .26 .89 .03 .573 .576 .0)^{T}$ \\
\hline
\end{tabular}

Table 2. The MSA and RMSE for joint-space controllers.

transmission. However, the results are not perfect, and they show that the proposed method has a higher variability of the $C$ values compared to both human data and earlier results obtained on a 3 -link arm without delays [4,39]. The data analysis suggests that this difference might be related to the need for better tuning of the architectural 


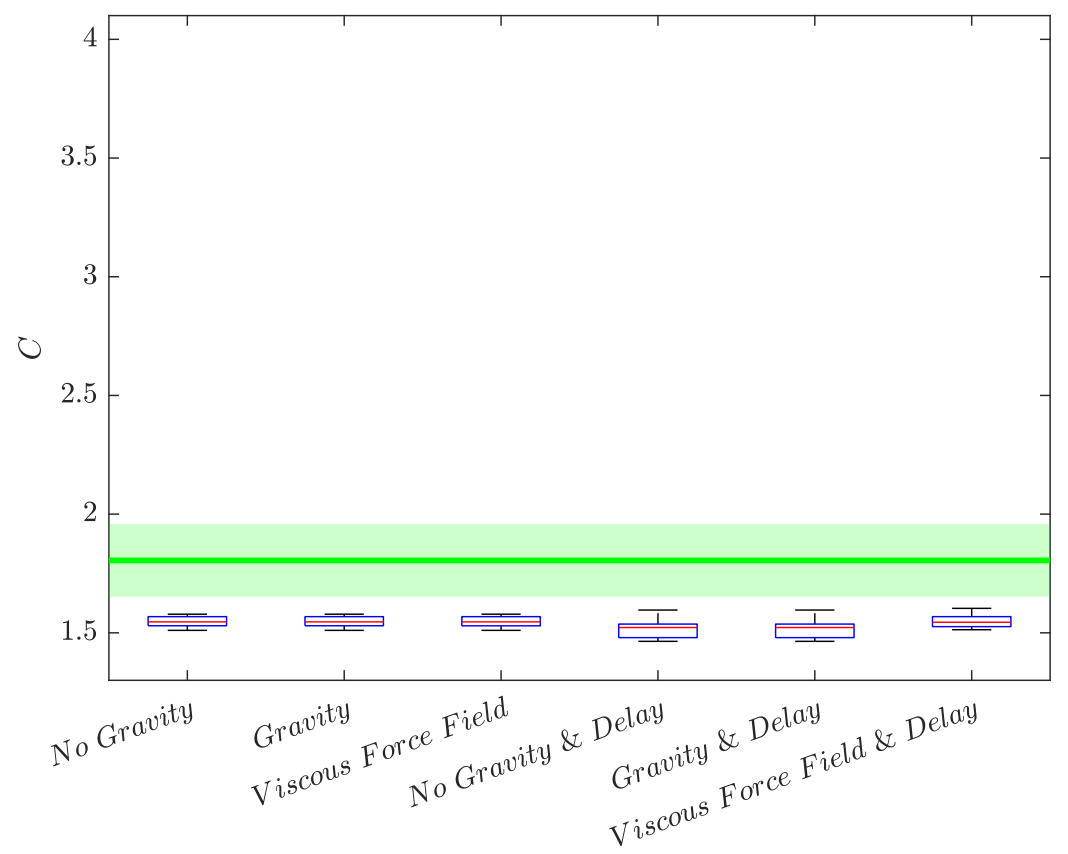

Figure 4. The $C$ value of the planned trajectories is consistent with harmonic trajectories $\left(C_{\mathrm{HT}}=1.596\right)$ [4], which is significantly lower compared to the human values of $C_{\mathrm{H}}=1.805 \pm 0.153[39,45$, reported in green.

parameters and better coordination in issuing the motor command. Additionally, the analysis tracking errors reported in Tables I and II indicate that a state estimator is needed to reduce the accumulation of error which increases the errors for $\tilde{x}_{d}$ beyond the selected task's accuracy, set with $\tilde{x}_{\mathrm{b}}$.

Nevertheless, these issues mentioned above are beyond the scope of this manuscript, which focuses on the analysis of the feasibility and the stability of the proposed method. Consequently, we will continue to further investigate methods which can improve the coordination between the different architectural components and reduce systematic errors. It is worth mentioning that the data is compared against the data of young, healthy individuals [39] during the execution of a trivial reaching task. Therefore, these human subjects can be assumed to be at the best of their performances considering the health and their motor training. In fact, research data comparing hand trajectories post-stroke and healthy subjects show how the number and relative magnitude of the velocity peaks (i.e., sub-movements) are correlated with the pathological severity 46].

The performance of the H-PMP indicates that the proposed method is capable of robustly generalising movements across different environmental dynamics, and it can induce human-like movements without regression from human data. The H-PMP integrates into a single computationally inexpensive architecture the task-separation principle, the uncontrolled manifold hypothesis, and the EPH without using projected dynamics and non-linear optimisation. In contrast, the $\lambda_{0}-\mathrm{PMP}$ and the OFC rely on numerical optimisation and projected dynamics, meaning their optimised strategies couple the system and the task dynamics, affecting the generality of the solution. 


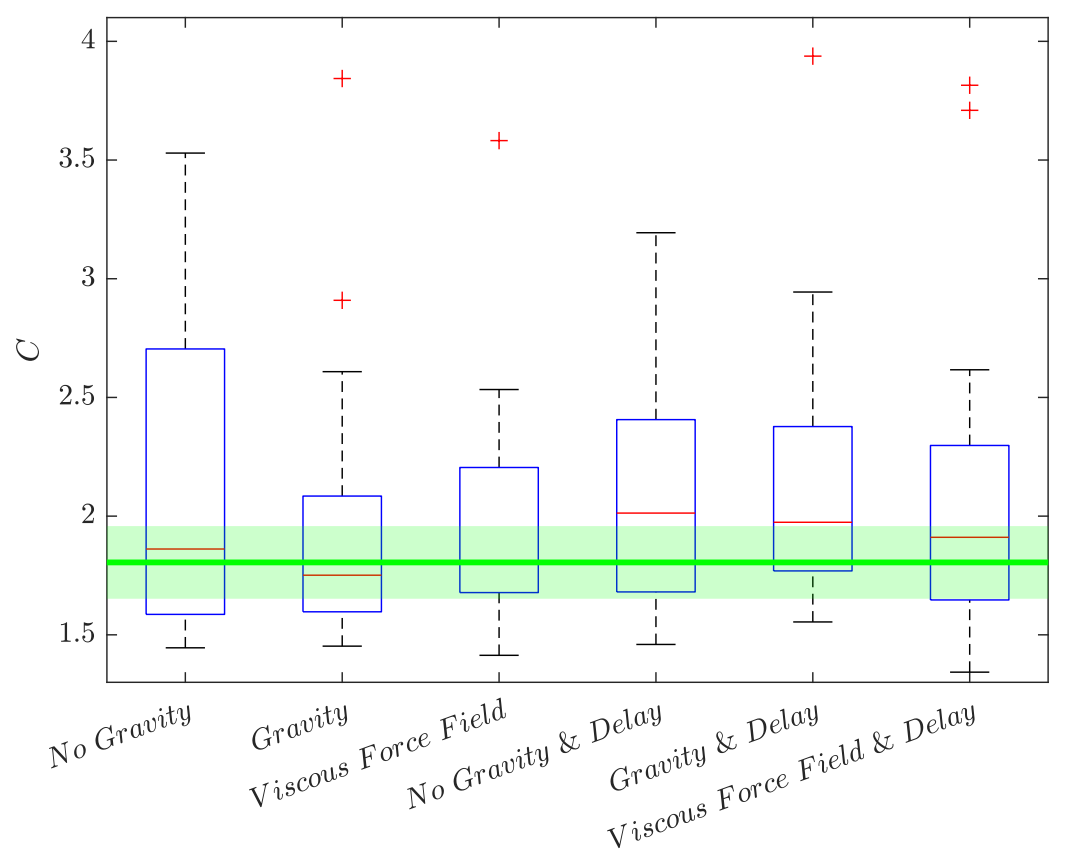

Figure 5. The $C$ value of the executed trajectories increases compared to the values of the planner trajectories. The data show high variability of the values probably connected to velocity peaks due to local perturbation, considering the tracking errors reported in Table 1. The human values of $C_{\mathrm{H}}=1.805 \pm 0.153$, [39], are reported in green, and their mean value is contained within the standard deviation in all the experimental conditions.

The H-PMP is composed of a series of hierarchically semi-autonomous controllers and planner and a deterministic non-linear mapping for postural optimisation robust to singularities [5, 42]. The planners and controller are stable non-linear oscillators (i.e., fractal attractor) that can be tuned online, and are robust to delays and model uncertainties [4, 5, 9, 10, 41]. Moreover, it is also an algorithmic representation of a Liénard system (i.e., Van der Pol oscillators and CPG) [5], which have been observed in biological systems $2,24,36]$. The non-linear mapping used for the postural optimisation (Equation 3 and Equation 4) solves the static component of the task-separation principle, while the Task-Space Interaction Controllers (Equation 7) account for the dynamic interaction. This implies there exists a deterministic solution to the task separation principle that can be learned by neural networks generating non-linear maps. Additionally, motor strategies could be classified via the affinity of the network input state, which agrees with the experimental observation of the motor synergies [47, 48. These two characteristics are also compatible with the Adaptive Resonance Theory (ART), which is a neural network framework capable of explaining how the nervous system learns, classifies and adapt in changing environmental conditions by building a non-linear representation of the knowledge [49].

In regards of the dynamic primitives, they are a concept originated in robotics to describe robot control strategies regressed by the desired task-space dynamics and 


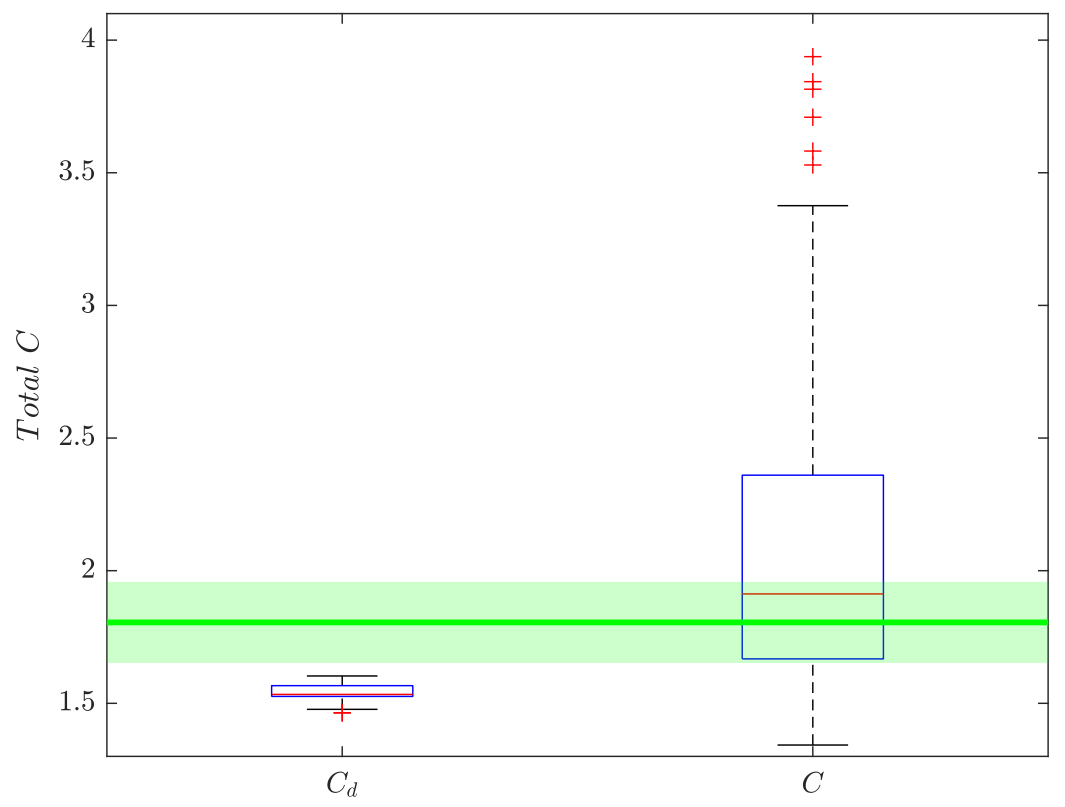

Figure 6. $C_{\mathrm{d}}$ are the values from the planner, $C$ indicates the values for the executed trajectories. The One-Way ANOVA confirmed that the $\mathrm{C}$ values of the measured trajectories from the different experimental condition are from the same population $(p=0.9442)$. The human values of $C_{\mathrm{H}}=1.805 \pm 0.153$, [39], are reported in green. Confirming a substantial overlap of the trajectories distributions.

recently borrowed by computational neuroscience to explain motor primitives in the contest of dynamic interaction $[1,2,14,17,18]$. This parallel has been suggested based on the observation that humans synchronise with external dynamics [3, 47, 50], and the dynamic primitives provide a model-based method to regress the interaction with the external dynamics [1,51]. However, this also implies that the motor primitive stability and accuracy depend on the quality of the environmental dynamics model, and they are difficult to generalise due to the coupling of the body and environmental dynamics. An equivalent form of regression of the control law from the desired taskspace dynamics to solve the task-separation principle is performed by both the OFC and the $\lambda_{0}$-PMP via numerical optimisation and projected dynamics [19, 27]. In contrast, the H-PMP deterministic mapping suggests that the dynamics primitives can be explained by exploiting the postural optimisation to achieve the desired alignment of the limb mechanical property with the principal direction of the environmental attractor. Meanwhile, trajectory desired velocity and limb stiffness modulation can be used to achieve synchronisations and desired reluctance to perturbation, respectively. This interpretation is supported by the data collected for studying how humans learn external dynamics, which shows that task performance increases with the synchronisation of the human strategy with the attractor of the external dynamics [3, 47, 52 55]. The process can be divided into three subtasks: 1) Understanding the direction of the energy flow (i.e., attractor principal directions). 2) Synchronisation with the autonomous dynamics (i.e., getting the timing right). 3) Control the tracking accuracy (i.e., impedance 

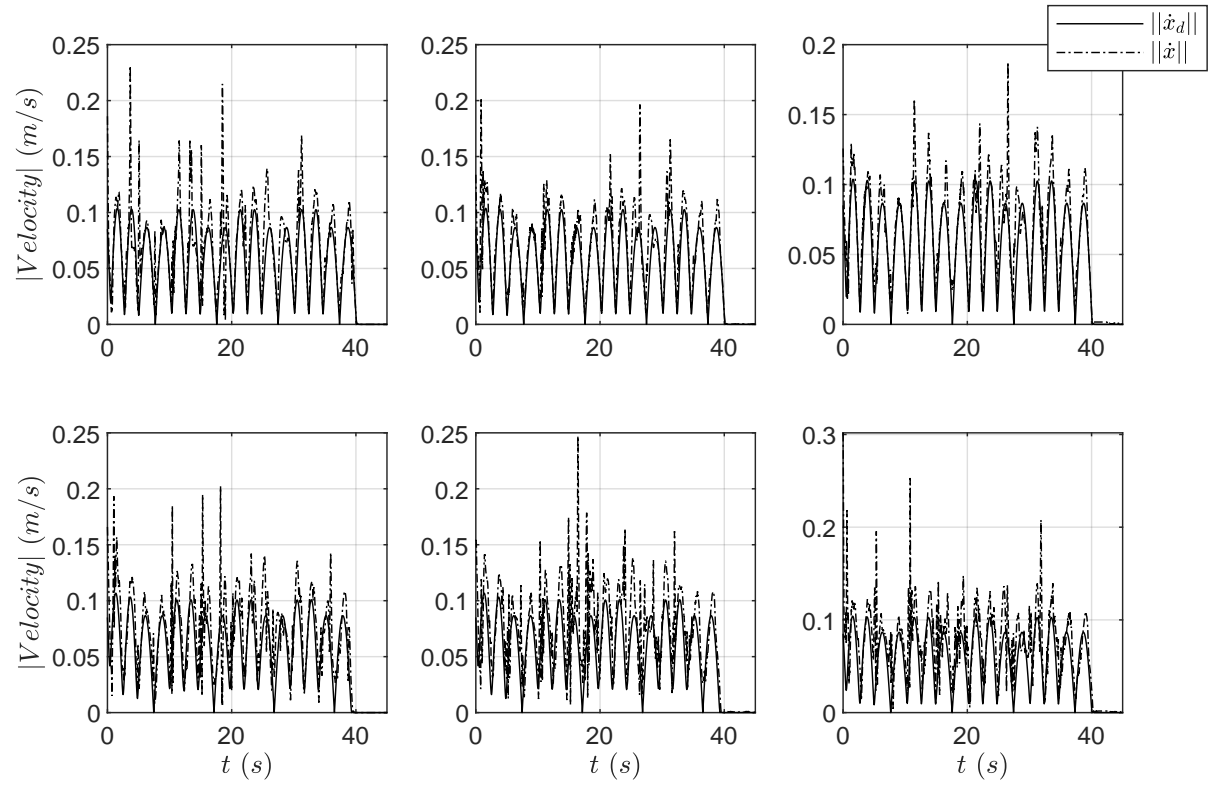

Figure 7. Comparing the absolute values of the desired and measured end-effector tangential velocities in the six experiments in Figure 3. The data from the experiment without delay are on the top row, the No Gravity experiment is on the left, the experiments with just gravity are on the centre, and the viscous force field results are reported on the right. The data show the characteristic bell-shaped profile; however, the measured trajectories show sporadic peaks that are probably generated by a combination of local perturbation and the limitations in motion coordination introduced by fixing the controller parameters.

modulation). It should be noted that these are the same experiment, which used to justify the regression-based approach mentioned above.

\section{Conclusion}

The H-PMP explains how the nervous system can robustly control the human body in challenging dynamic conditions despite the substantial information delays. The model achieves these results without any formal modelling of either the limb and the environment dynamics. In the future, the architecture's performance could be further improved by introducing the haptic feedback, the state estimator and learning algorithms for the online tuning and synchronisation of the architecture planner and controllers.

\section{Acknowledgements}

This work has been supported by EPSRC UK RAI Hub ORCA (EP/R026173/1), National Centre for Nuclear Robotics (NCNR EPR02572X/1) and THING project in the EU Horizon 2020 (ICT-2017-1). 


\section{References}

[1] G. Averta and N. Hogan, "Enhancing robot-environment physical interaction via optimal impedance profiles," in 2020 8th IEEE RAS/EMBS International Conference for Biomedical Robotics and Biomechatronics (BioRob), 2020, pp. 973-980.

[2] C. Tiseo, "Modelling of bipedal locomotion for the development of a compliant pelvic interface between human and a balance assistant robot," Doctoral thesis, 2018.

[3] C. Tiseo, K. Veluvolu, and W. Ang, "The bipedal saddle space: modelling and validation," Bioinspiration \& biomimetics, vol. 14, no. 1, p. 015001, 2018.

[4] C. Tiseo, S. R. Charitos, and M. Mistry, "Theoretical evidence supporting harmonic reaching trajectories," in 2021 10th International IEEE/EMBS Conference on Neural Engineering (NER). IEEE, 2021.

[5] — - "Exploiting spherical projections to generate human-like wrist pointing movements," in 2021 43rd Annual International Conference of the IEEE Engineering in Medicine and Biology Society $(E M B C), 2021$.

[6] G. Xin, W. Wolfslag, H.-C. Lin, C. Tiseo, and M. Mistry, "An optimization-based locomotion controller for quadruped robots leveraging cartesian impedance control," Frontiers in Robotics and $A I$, vol. 7, p. $48,2020$.

[7] C. Mastalli, R. Budhiraja, W. Merkt, G. Saurel, B. Hammoud, M. Naveau, J. Carpentier, L. Righetti, S. Vijayakumar, and N. Mansard, "Crocoddyl: An efficient and versatile framework for multi-contact optimal control," in 2020 IEEE International Conference on Robotics and Automation (ICRA), 2020, pp. 2536-2542.

[8] H. Ferrolho, W. Merkt, V. Ivan, W. Wolfslag, and S. Vijayakumar, "Optimizing dynamic trajectories for robustness to disturbances using polytopic projections," in 2020 IEEE/RSJ International Conference on Intelligent Robots and Systems (IROS), 2020, pp. 7477-7484.

[9] K. K. Babarahmati, C. Tiseo, J. Smith, H. C. Lin, M. S. Erden, and M. Mistry, "Fractal impedance for passive controllers," arXiv preprint arXiv:1911.04788, 2019.

[10] K. K. Babarahmati, C. Tiseo, Q. Rouxel, Z. Li, and M. Mistry, "Robust high-transparency haptic exploration for dexterous telemanipulation," in Proc. IEEE International Conference on Robotics and Automation (ICRA), 2021.

[11] C. Tiseo, W. Merkt, K. K. Babarahmati, W. Wolfslag, S. Vijayakumar, and M. Mistry, "Biomimetic adaptive force/position control using fractal impedance," in 2020 8th IEEE RAS/EMBS International Conference for Biomedical Robotics and Biomechatronics (BioRob), 2020, pp. $1180-1187$.

[12] N. Hogan and S. P. Buerger, "Impedance and interaction control," in Robotics and automation handbook. CRC press, 2018, pp. 375-398.

[13] C. Tiseo, V. Ivan, W. Merkt, I. Havoutis, M. Mistry, and S. Vijayakumar, "A passive navigation planning algorithm for collision-free control of mobile robots," in Proc. IEEE International Conference on Robotics and Automation (ICRA), 2021.

[14] A. J. Ijspeert, J. Nakanishi, H. Hoffmann, P. Pastor, and S. Schaal, "Dynamical movement primitives: learning attractor models for motor behaviors," Neural computation, vol. 25, no. 2, pp. 328-373, 2013.

[15] T. Flash and B. Hochner, "Motor primitives in vertebrates and invertebrates," Current opinion in neurobiology, vol. 15, no. 6, pp. 660-666, 2005.

[16] D. Mitrovic, S. Klanke, and S. Vijayakumar, "Adaptive optimal feedback control with learned internal dynamics models," in From Motor Learning to Interaction Learning in Robots. Springer, 2010, pp. 65-84.

[17] N. Hogan and D. Sternad, "Dynamic primitives of motor behavior," Biological cybernetics, vol. 106, no. 11, pp. 727-739, 2012.

[18] — - "Dynamic primitives in the control of locomotion," Frontiers in computational neuroscience, vol. 7 , p. 71, 2013. 
[19] P. Tommasino and D. Campolo, "An extended passive motion paradigm for human-like posture and movement planning in redundant manipulators," Frontiers in neurorobotics, vol. 11, p. 65, 2017.

[20] J. Ahn and N. Hogan, "Walking is not like reaching: evidence from periodic mechanical perturbations," PloS one, vol. 7, no. 3, p. e31767, 2012.

[21] R. Shadmehr and S. P. Wise, The computational neurobiology of reaching and pointing: a foundation for motor learning. MIT press, 2005.

[22] J. G. Milton, "The delayed and noisy nervous system: implications for neural control," Journal of Neural Engineering, vol. 8, no. 6, p. 065005, oct 2011.

[23] G. Avraham, E. Sulimani, F. A. Mussa-Ivaldi, and I. Nisky, "Effects of visuomotor delays on the control of movement and on perceptual localization in the presence and absence of visual targets," Journal of neurophysiology, vol. 122, no. 6, pp. 2259-2271, 2019.

[24] S. P. Wise and R. Shadmehr, "Motor Control," in Encyclopedia of the Human Brain. Elsevier, 2002, vol. 3, pp. 137-157. [Online]. Available: http://linkinghub.elsevier.com/retrieve/pii/B0122272102002168

[25] E. Todorov, "Direct cortical control of muscle activation in voluntary arm movements: A model," Nature Neuroscience, vol. 3, no. 4, pp. 391-398, 2000.

[26] E. Todorov and M. I. Jordan, "Optimal feedback control as a theory of motor coordination," Nature neuroscience, vol. 5, no. 11, pp. 1226-1235, 2002.

[27] E. Guigon, P. Baraduc, and M. Desmurget, "Computational motor control: redundancy and invariance," Journal of neurophysiology, vol. 97, no. 1, pp. 331-347, 2007.

[28] V. Mohan and P. Morasso, "Passive motion paradigm: an alternative to optimal control," Frontiers in Neurorobotics, vol. 5, p. 4, 2011.

[29] P. Tommasino and D. Campolo, "Task-space separation principle: a force-field approach to motion planning for redundant manipulators," Bioinspiration \&3 biomimetics, vol. 12, no. 2, p. 026003, 2017.

[30] S. H. Scott, "Optimal feedback control and the neural basis of volitional motor control," Nature Reviews Neuroscience, vol. 5, no. 7, pp. 532-545, 2004.

[31] R. Shadmehr and J. W. Krakauer, "A computational neuroanatomy for motor control," Experimental brain research, vol. 185, no. 3, pp. 359-381, 2008.

[32] B. E. Maki and W. E. McIlroy, "Cognitive demands and cortical control of human balance-recovery reactions," Journal of neural transmission, vol. 114, no. 10, pp. 1279-1296, 2007.

[33] C. Tiseo and W. T. Ang, "The balance: An energy management task," in 2016 6th IEEE International Conference on Biomedical Robotics and Biomechatronics (BioRob). IEEE, 2016, pp. 723-728.

[34] C. Haridas, E. P. Zehr, and J. E. Misiaszek, "Postural uncertainty leads to dynamic control of cutaneous reflexes from the foot during human walking," Brain research, vol. 1062, no. 1-2, pp. $48-62,2005$.

[35] M. Pijnappels, M. F. Bobbert, and J. H. van Dieën, "How early reactions in the support limb contribute to balance recovery after tripping," Journal of Biomechanics, vol. 38, no. 3, pp. 627-634, mar 2005. [Online]. Available: http://linkinghub.elsevier.com/retrieve/pii/S0021929004001708

[36] Y. P. Ivanenko, R. E. Poppele, and F. Lacquaniti, "Motor Control Programs and Walking," The Neuroscientist, vol. 12, no. 4, pp. 339-348, aug 2006. [Online]. Available: http://nro.sagepub.com/cgi/doi/10.1177/1073858406287987

[37] D. Bucher, G. Haspel, J. Golowasch, and F. Nadim, "Central Pattern Generators," in eLS. Chichester, UK: John Wiley \& Sons, Ltd, dec 2015, pp. 1-12. [Online]. Available: http://doi.wiley.com/10.1002/9780470015902.a0000032.pub2

[38] J. Diedrichsen, R. Shadmehr, and R. B. Ivry, "The coordination of movement: optimal feedback control and beyond," Trends in cognitive sciences, vol. 14, no. 1, pp. 31-39, 2010.

[39] T. Flash and N. Hogan, "The coordination of arm movements: an experimentally confirmed 
mathematical model," Journal of neuroscience, vol. 5, no. 7, pp. 1688-1703, 1985.

[40] C. Tiseo, W. Merkt, W. Wolfslag, S. Vijayakumar, and M. Mistry, "Safe and compliant control of redundant robots using superimposition of passive task-space controllers," arXiv preprint arXiv:2002.12249, 2020.

[41] C. Tiseo, W. Merkt, K. K. Babarahmati, W. Wolfslag, I. Havoutis, S. Vijayakumar, and M. Mistry, "Hapfic: An adaptive force/position controller for safe environment interaction in articulated systems," IEEE Transactions on Neural Systems and Rehabilitation Engineering, pp. 1-1, 2021.

[42] T. Tiseo, S. R. Charitos, and M. Mistry, "Geometrical postural optimisation of 7-dof limb-like manipulators," arXiv preprint arXiv:2107.02715, 2021.

[43] N. Hogan, "Adaptive control of mechanical impedance by coactivation of antagonist muscles," IEEE Transactions on automatic control, vol. 29, no. 8, pp. 681-690, 1984.

[44] D. Campolo, F. Widjaja, M. Esmaeili, and E. Burdet, "Pointing with the wrist: a postural model for donders' law," Experimental brain research, vol. 212, no. 3, pp. 417-427, 2011.

[45] M. J. Richardson and T. Flash, "Comparing smooth arm movements with the two-thirds power law and the related segmented-control hypothesis," Journal of neuroscience, vol. 22, no. 18, pp. 8201-8211, 2002.

[46] M. A. Murphy, C. Willén, and K. S. Sunnerhagen, "Kinematic variables quantifying upper-extremity performance after stroke during reaching and drinking from a glass," Neurorehabilitation and neural repair, vol. 25, no. 1, pp. 71-80, 2011.

[47] S. Bazzi, J. Ebert, N. Hogan, and D. Sternad, "Stability and predictability in human control of complex objects," Chaos: An Interdisciplinary Journal of Nonlinear Science, vol. 28, no. 10, p. $103103,2018$.

[48] H. J. Chiel, L. H. Ting, Ö. Ekeberg, and M. J. Hartmann, "The brain in its body: motor control and sensing in a biomechanical context," Journal of Neuroscience, vol. 29, no. 41, pp. $12807-$ $12814,2009$.

[49] S. Grossberg, "Adaptive resonance theory: How a brain learns to consciously attend, learn, and recognize a changing world," Neural networks, vol. 37, pp. 1-47, 2013.

[50] S.-W. Park, H. Marino, S. K. Charles, D. Sternad, and N. Hogan, "Moving slowly is hard for humans: limitations of dynamic primitives," Journal of neurophysiology, vol. 118, no. 1, pp. 69-83, 2017.

[51] M. C. Nah, A. Krotov, M. Russo, D. Sternad, and N. Hogan, "Dynamic primitives facilitate manipulating a whip," in 2020 8th IEEE RAS/EMBS International Conference for Biomedical Robotics and Biomechatronics (BioRob). IEEE, 2020, pp. 685-691.

[52] S. Bazzi and D. Sternad, "Human control of complex objects: towards more dexterous robots," Advanced Robotics, vol. 34, no. 17, pp. 1137-1155, 2020.

[53] R. Nayeem, S. Bazzi, N. Hogan, and D. Sternad, "Transient behavior and predictability in manipulating complex objects," in 2020 IEEE International Conference on Robotics and Automation (ICRA). IEEE, 2020, pp. 10155-10161.

[54] P. Tommasino, A. Maselli, D. Campolo, F. Lacquaniti, and A. d'Avella, "A hessian-based decomposition characterizes how performance in complex motor skills depends on individual strategy and variability," BioRxiv, p. 645317, 2020.

[55] A. Maselli, A. Dhawan, M. Russo, B. Cesqui, F. Lacquaniti, and A. d'Avella, "A whole body characterization of individual strategies, gender differences, and common styles in overarm throwing," Journal of neurophysiology, vol. 122, no. 6, pp. 2486-2503, 2019. 